\title{
Lewis Y Antigen Binding
}

National Cancer Institute

\section{Source}

National Cancer Institute. Lewis Y Antigen Binding. NCI Thesaurus. Code C41554.

Lewis Y Antigen Binding involves non-covalent interaction of a molecule through intermolecular physical forces of attraction with the Lewis $Y$ cell surface antigen. 